\title{
Shading correction for cone-beam CT in radiotherapy: validation of dose calculation accuracy using clinical images
}

DOI:

10.1117/12.2254059

\section{Document Version}

Accepted author manuscript

Link to publication record in Manchester Research Explorer

Citation for published version (APA):

Marchant, T., Joshi, K., \& Moore, C. (2017). Shading correction for cone-beam CT in radiotherapy: validation of dose calculation accuracy using clinical images. In Proc. SPIE 10132, Medical Imaging 2017: Physics of Medical Imaging (Vol. 10132, pp. 101320J1-101320J11). [101320J] SPIE. https://doi.org/10.1117/12.2254059

\section{Published in:}

Proc. SPIE 10132, Medical Imaging 2017: Physics of Medical Imaging

\section{Citing this paper}

Please note that where the full-text provided on Manchester Research Explorer is the Author Accepted Manuscript or Proof version this may differ from the final Published version. If citing, it is advised that you check and use the publisher's definitive version.

\section{General rights}

Copyright and moral rights for the publications made accessible in the Research Explorer are retained by the authors and/or other copyright owners and it is a condition of accessing publications that users recognise and abide by the legal requirements associated with these rights.

\section{Takedown policy}

If you believe that this document breaches copyright please refer to the University of Manchester's Takedown Procedures [http://man.ac.uk/04Y6Bo] or contact uml.scholarlycommunications@manchester.ac.uk providing relevant details, so we can investigate your claim.

\section{OPEN ACCESS}




\title{
Shading correction for cone-beam CT in radiotherapy: Validation of dose calculation accuracy using clinical images
}

\author{
T E Marchant ${ }^{\mathrm{a}, \mathrm{b}}$, K D Joshi ${ }^{\mathrm{b}}$, and C J Moore ${ }^{\mathrm{a}, \mathrm{b}}$ \\ aThe University of Manchester, Manchester Academic Health Science Centre, The Christie \\ NHS Foundation Trust, Manchester, M20 4BX, UK. \\ ${ }^{\mathrm{b}}$ Christie Medical Physics and Engineering, The Christie NHS Foundation Trust, Manchester, \\ M20 4BX, UK.
}

\begin{abstract}
Cone-beam $\mathrm{CT}$ (CBCT) images are routinely acquired to verify patient position in radiotherapy (RT), but are typically not calibrated in Hounsfield Units (HU) and feature non-uniformity due to X-ray scatter and detector persistence effects. This prevents direct use of $\mathrm{CBCT}$ for re-calculation of RT delivered dose. We previously developed a prior-image based correction method to restore $\mathrm{HU}$ values and improve uniformity of CBCT images. Here we validate the accuracy with which corrected CBCT can be used for dosimetric assessment of RT delivery, using CBCT images and RT plans for 45 patients including pelvis, lung and head sites. Dose distributions were calculated based on each patient's original RT plan and using CBCT image values for tissue heterogeneity correction. Clinically relevant dose metrics were calculated (e.g. median and minimum target dose, maximum organ at risk dose). Accuracy of CBCT based dose metrics was determined using an "override ratio" method where the ratio of the dose metric to that calculated on a bulk-density assigned version of the image is assumed to be constant for each patient, allowing comparison to "gold standard" CT. For pelvis and head images the proportion of dose errors $>2 \%$ was reduced from $40 \%$ to $1.3 \%$ after applying shading correction. For lung images the proportion of dose errors $>3 \%$ was reduced from $66 \%$ to $2.2 \%$. Application of shading correction to CBCT images greatly improves their utility for dosimetric assessment of RT delivery, allowing high confidence that $\mathrm{CBCT}$ dose calculations are accurate within $2-3 \%$.
\end{abstract}

Keywords: CLIN (clinical evaluation), CTCB (cone-beam CT), ONC (oncology)

This paper was presented at SPIE Medical Imaging 2017:

T Marchant, K Joshi, C Moore, "Shading correction for cone-beam CT in radiotherapy: Validation of dose calculation accuracy using clinical images," Medical Imaging 2017: Physics of Medical Imaging, Proc. SPIE 10132, 101320J (2017).

http://dx.doi.org/10.1117/12.2254059

Copyright 2017 Society of Photo Optical Instrumentation Engineers. One print or electronic copy may be made for personal use only. Systematic electronic or print reproduction and distribution, duplication of any material in this paper for a fee or for commercial purposes, or modification of the content of the paper are prohibited.

\section{INTRODUCTION}

Cone-beam $\mathrm{CT}(\mathrm{CBCT})$ images are routinely acquired to verify patient position in radiotherapy $(\mathrm{RT})^{1}$. However CBCT images are typically not calibrated in Hounsfield Units (HU) and suffer from non-uniformity due to X-ray scatter, patient motion and detector persistence effects ${ }^{2-4}$. This means the tissue densities from CBCT cannot be used directly for re-calculation of RT delivered dose based on the image of the day.

Various methods have been introduced to enable radiotherapy dose calculations based on CBCT images. These include scatter corrections ${ }^{5,6}$, deformable registration with planning $\mathrm{CT}^{7-9}$, and uniformity corrections incorporating prior-information either in projection space ${ }^{10}$ or reconstructed image space ${ }^{11}$.

Corresponding author: tom.marchant@christie.nhs.uk 
We previously developed a prior-image based correction method to restore HU values and improve uniformity of $\mathrm{CBCT}$ images ${ }^{12}$. Here we validate the accuracy with which corrected CBCT can be used for dosimetric assessment of RT delivery, based on a large sample of clinical images at three different anatomical sites.

\section{METHODS}

CBCT images and RT plan data were collected for 45 patients undergoing radiotherapy (15 pelvis, 15 head and neck (H\&N), 15 lung). Three CBCT images per patient were included in the study (135 CBCT images total). CBCT images were acquired with the Elekta XVI linac-integrated CBCT system (Elekta AB, Stockholm, Sweden), using XVI versions 4.5 or 5.0. All images were acquired routinely as part of the patients' radiotherapy, and the study was performed retrospectively. All image and RT plan data was anonymized and ethical approval was obtained for inclusion in the study (UK REC ref. 15/LO/0146).

\subsection{Shading correction algorithm}

All CBCT images were processed to restore HU values and remove non-uniformity artifacts using an updated and optimized version of a previously reported correction algorithm ${ }^{12}$, referred to here as the "shading correction". The correction uses prior information from a high-quality, fan-beam CT image of the patient acquired prior to treatment for use in RT planning. First the image histograms of the CT and CBCT images are compared to derive a linear scaling to be applied to the CBCT image which best matches the main histogram peak positions (air and soft tissue) to the $\mathrm{CT}$ image. The CT image is then rigidly registered to the $\mathrm{CBCT}$ and resampled onto the CBCT pixel matrix. Next regions representing soft-tissue are segmented in both images and all other regions (e.g. air, lung, bone) are removed before being filled by interpolation from surrounding soft-tissue areas. The CBCT image is then divided by the CT image to create a "shading map" indicating the relative brightness of each pixel in the CBCT image compared to the CT. Finally the shading map is smoothed so that it retains only low frequency variations in image brightness, before being applied to the CBCT image as a correction map to produce the final corrected image. Figure 1 shows an example pelvis CBCT image before and after application of the shading correction. Modifications to the correction algorithm used in this work include reimplementing it completely in $\mathrm{C}++$, using the Insight Toolkit (ITK) for image registration and segmentation tasks; optimising the histogram scaling part of the algorithm to remove the need for hard-coded image-specific parameters; adding additional processing steps to allow the processing of lung images.

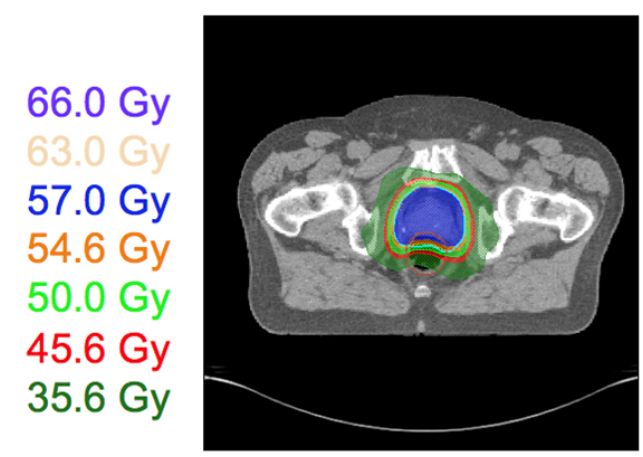

CT

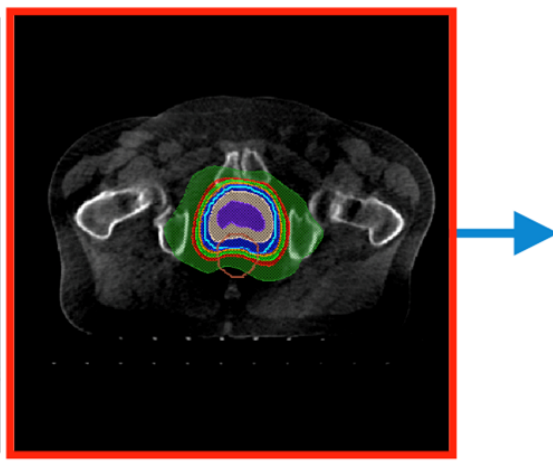

CBCT

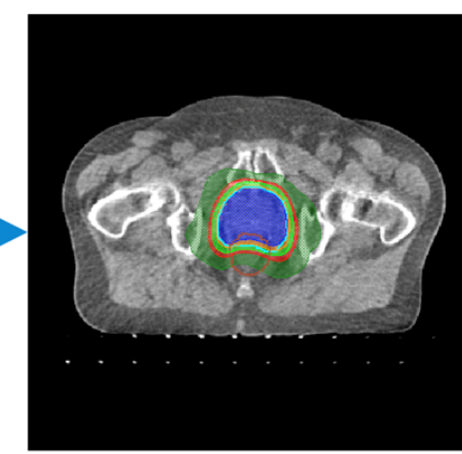

Corrected СВСТ

Figure 1. Pelvis CT and CBCT images before and after application of the shading correction (all displayed with window level 0, width 600). RT dose distributions are overlaid to illustrate the effect of using raw CBCT image values for heterogeneity correction.

\subsection{CBCT based dosimetric assessment of RT delivery}

All images were imported to the Pinnacle RT planning system (Philips Radiation Oncology Systems, Fitchburg, WI, USA), where the patient's original RT plan was used to recalculate the RT dose distribution using the CBCT image values as the basis for heterogeneity correction (taking into account X-ray attenuation properties 
of tissues with different density). Further details of the treatment sites and treatment plans used are given in table 1. CBCT images were only included in the study if the field of view (FOV) was sufficient to calculate dose in all relevant areas. Usually this means that the FOV should encompass the patient outline at all points where it is traversed by the radiotherapy beams. This requirement is satisfied for the majority of pelvis or thorax patients imaged with Medium or Large FOV (41 cm or $50 \mathrm{~cm}$ diameter). H\&N patients are typically imaged with Small FOV $(26 \mathrm{~cm})$ which is not large enough to encompass the patient surface below the level of the shoulders. Hence it is not possible to calculate dose based on the CBCT image in this region unless some method is used to re-create the missing patient surface position. In this study we chose to only evaluate dose to the primary disease target volume for $\mathrm{H} \& \mathrm{~N}$ patients, and did not consider dose to any nodal target volume that may extend below the level of the shoulders.

Table 1: Radiotherapy plan characteristics for validation of CBCT based dose calculations. Abbreviations: 3D conformal radiotherapy (3DCRT), Volumetric modulated arc therapy (VMAT), Intensity modulated radiotherapy (IMRT), Stereotactic ablative body radiotherapy (SABR).

\begin{tabular}{|c|c|c|c|c|c|c|}
\hline & & Technique & & & & \\
\hline Site & Patients & & Beams/arcs & Target Dose & Fractions & Energy \\
\hline Bladder & 5 & 3DCRT & $4-5$ & 24-52 Gy & $12-20$ & $8 \mathrm{MV}$ \\
\hline Prostate & 9 & VMAT & 1 & $60 \mathrm{~Gy}$ & 20 & 8-10MV \\
\hline Prostate & 1 & IMRT & 5 & $60 \mathrm{~Gy}$ & 20 & $8 \mathrm{MV}$ \\
\hline Brain & 1 & 3DCRT & 3 & $50 \mathrm{~Gy}$ & 30 & $6 \mathrm{MV}$ \\
\hline $\begin{array}{l}\text { Pharynx, Oral cavity, } \\
\text { Parotid, Tonsil }\end{array}$ & 14 & VMAT & 2 & 50-66 Gy & $20-30$ & $6 \mathrm{MV}$ \\
\hline Lung & 2 & 3DCRT & 3 & 55-57 Gy & 20 & $6 \mathrm{MV}$ \\
\hline Lung & 11 & IMRT & $4-7$ & 50-66 Gy & $20-33$ & $6 \mathrm{MV}$ \\
\hline Lung & 2 & SABR & 8 & $60 \mathrm{~Gy}$ & 5 & $6 \mathrm{MV}$ \\
\hline
\end{tabular}

Three clinically relevant dose metrics were calculated for each plan: median or mean dose to Planning Target Volume (PTV), Dose covering $99 \%$ or $95 \%$ of PTV ( $\mathrm{D}_{99}$ or $\mathrm{D}_{95}$ ) or minimum dose to 1cc of PTV, and organ at risk (OAR) dose metric(s) relevant to the anatomic site: volume of rectum receiving greater than 40Gy $\left(\mathrm{V}_{40 \mathrm{~Gy}}\right)$ for pelvis plans, maximum dose to spinal cord (SC) for lung plans, contralateral parotid mean dose and maximum dose to SC for H\&N plans.

\subsection{Override ratio validation method}

Because of anatomical changes between the planning CT and CBCT images, it is not expected that the dose metric calculated on CBCT should be identical to that calculated on CT. Instead we repeat the dose calculation after applying bulk density overrides to the image, and calculate the ratio of the dose metric before and after applying the density override (the "override ratio"). We expect that this ratio should remain constant for a particular patient and RT plan. This is because the error introduced by applying the bulk override depends mainly on the individual patient's tissue densities and the path length through different tissues traversed by each beam, factors that will remain approximately constant for a particular patient/plan. Hence the errors in CBCT-based dose calculation can be revealed by comparing the CBCT override-ratio to that calculated from the planning CT image (the gold standard). For pelvis and H\&N images all tissue within the body contour was assigned density of $1 \mathrm{gcm}^{3}$. For lung images the lung tissue was also assigned a uniform density based on the mean lung HU for that patient. For pelvis images, any regions of gas in the rectum or bowel were also overridden with a density of $1 \mathrm{gcm}^{3}$ when calculating dose using heterogeneity correction based on the image values. This was to avoid the effect that changing pockets of gas may have on constancy of the override ratio between CBCT and CT.

The procedure is illustrated in figure 2, where the override ratio for a dose metric calculated from CBCT $(\mathrm{C} / \mathrm{D})$ should equal that calculated from $\mathrm{CT}(\mathrm{A} / \mathrm{B})$. 
The override-ratio method for determining CBCT dose calculation errors was validated using 5 patients with repeat planning CT (4 lung cases and 1 pelvis case). The override-ratio was calculated for several target and OAR dose metrics for both CT images for each patient. This allowed us to test the assumption that the override-ratio remains constant for a particular patient and radiotherapy plan. Patients having repeat planning CT will tend to be those where anatomical changes were observed during their radiotherapy. Hence this sample, while small, should test the robustness of the override-ratio method to significant changes between images.

\section{Calculate dose on CT image}

\section{Calculate dose on CBCT image}

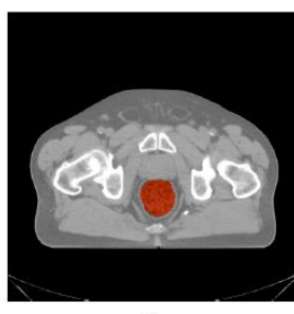

A

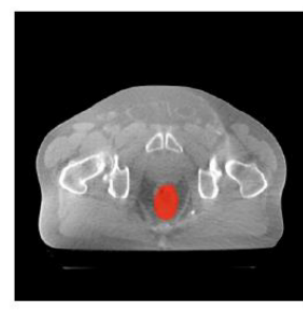

$C$

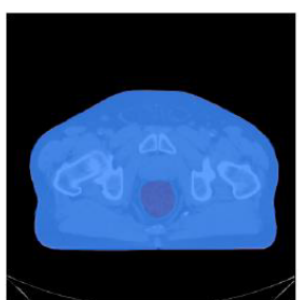

B

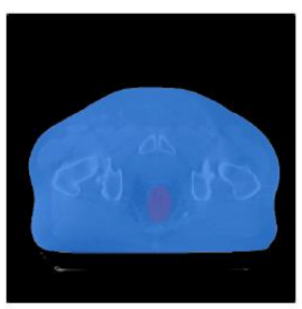

D
Calculate dose on

CT image with body density

override

Calculate dose on

CBCT image with body density

override

Figure 2. Override ratio method to determine errors in dose metrics calculated from CBCT.

\section{RESULTS}

\subsection{Validation of override ratio method}

For the five cases with repeat planning CT the override-ratio for the second CT was within $2 \%$ of the first CT for all of the dose metrics tested. The mean difference was $0.5 \%$ with standard deviation $0.7 \%$. Figure $3 \mathrm{a}$ shows a histogram of the differences in override-ratio between the two CT images. It should be noted that some of the images used in this validation showed quite large anatomical differences between the two planning CT images. For example, figure 3b-c shows original and repeat CT images for one of the lung patients where there is a significant difference in lung volume, and tumour size. As a result of anatomical changes, the dose metrics tested were not always similar between the two images. Four out of five patients had at least one metric differing by more than $3 \%$ between the two images, with a maximum difference observed of $39 \%$. The presence of these differences demonstrates that similar override-ratio values are obtained for two images of the same patient even when there are significant anatomical changes between the images (as may be the case during a course of radiotherapy).

\subsection{Pelvis images}

Figure 4 shows the estimated errors for each of the dose metrics assessed for pelvis patients, for raw (uncorrected) $\mathrm{CBCT}$ and after applying the shading correction. The mean error and spread of errors is greatly improved for corrected CBCT, with nearly all errors less than $2 \%$ for the corrected images. Table 2 summarises the median and standard deviation of dose errors for each of the dose metrics used for pelvis plans. The percentage of dose metric errors greater than $2 \%$ and $3 \%$ is also shown. 
(a)

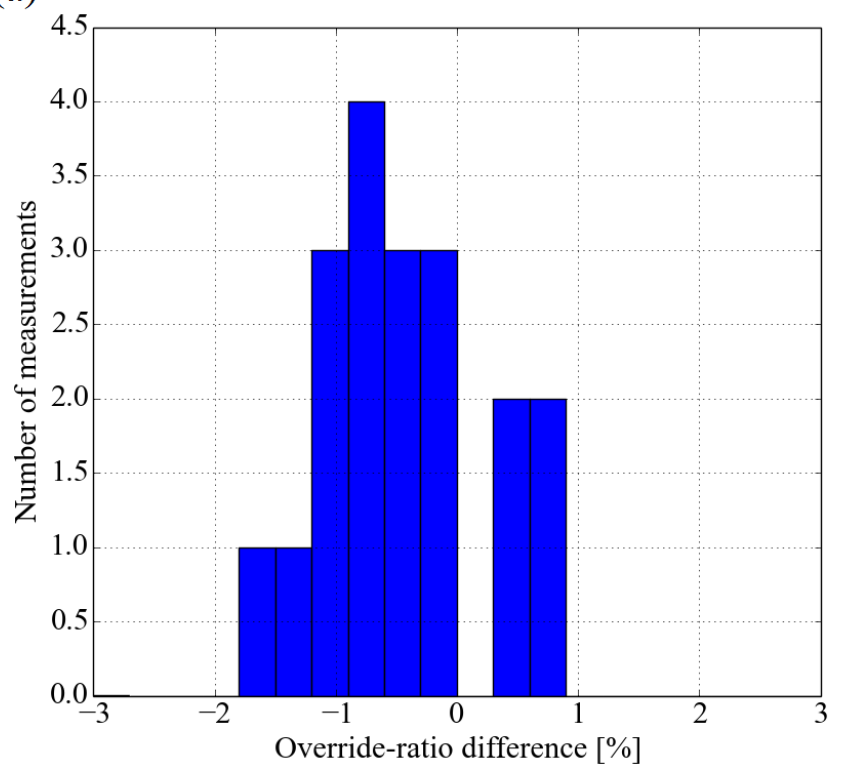

(b)
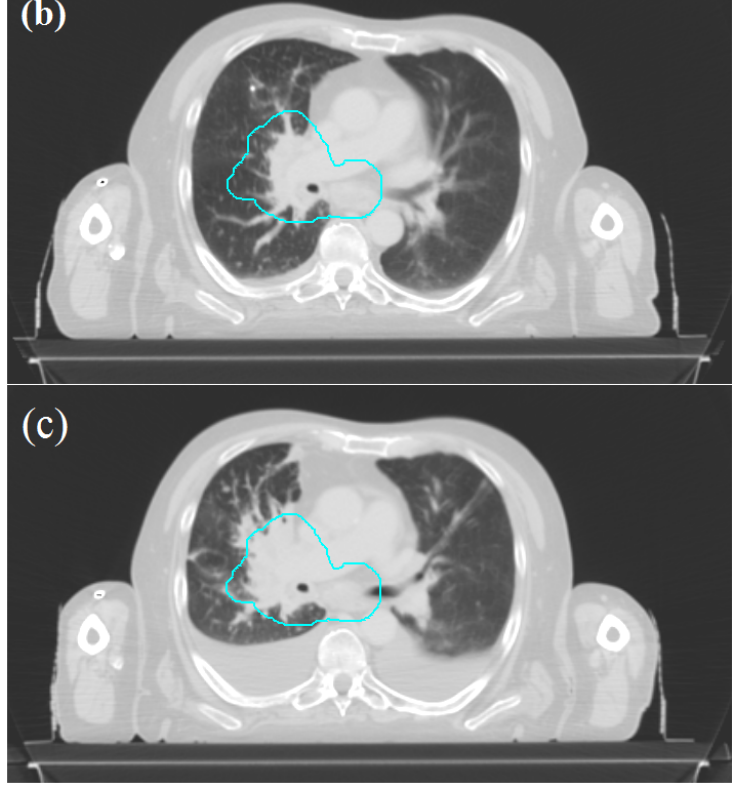

Figure 3. (a) Histogram showing differences between override-ratios calculated for two CT images of the same patient. (b \& c) Lung case showing significant change between original (b) and repeat (c) planning CT images. Radiotherapy planning target volume (PTV) is shown in light blue.

Table 2: Summary of results for pelvis dose metrics.

\begin{tabular}{llrrrr}
\hline & Metric & Median err & SD err & Err $<2 \%$ & Err $<3 \%$ \\
\hline \multirow{3}{*}{ Raw } & PTV median & $7.92 \%$ & $2.48 \%$ & $13.3 \%$ & $13.3 \%$ \\
CBCT & PTV D99 & $7.58 \%$ & $2.43 \%$ & $13.3 \%$ & $13.3 \%$ \\
& Rectum V40.8 & $9.15 \%$ & $5.45 \%$ & $20.0 \%$ & $20.0 \%$ \\
& Combined & $7.91 \%$ & $3.83 \%$ & $15.6 \%$ & $15.6 \%$ \\
\hline \multirow{3}{*}{ Corrected } & PTV median & $-0.18 \%$ & $0.25 \%$ & $100.0 \%$ & $100.0 \%$ \\
CBCT & PTV D99 & $-0.20 \%$ & $0.21 \%$ & $100.0 \%$ & $100.0 \%$ \\
& Rectum V40.8 & $-0.57 \%$ & $0.70 \%$ & $95.6 \%$ & $97.8 \%$ \\
& Combined & $-0.24 \%$ & $0.48 \%$ & $98.5 \%$ & $99.3 \%$ \\
\hline
\end{tabular}

\section{3 $\mathrm{H} \& \mathrm{~N}$ images}

Figure 5 shows the estimated errors for each of the dose metrics assessed for H\&N patients. Again the mean error and spread of errors is reduced for shading corrected CBCT. However for H\&N images the errors for raw CBCT are already quite small, so the improvement is not as large as for pelvis images. See section 4 for further discussion of this. Table 3 summarises the median and standard deviation of dose errors for each of the dose metrics used for H\&N plans. The percentage of dose metric errors greater than $2 \%$ and $3 \%$ is also shown. 

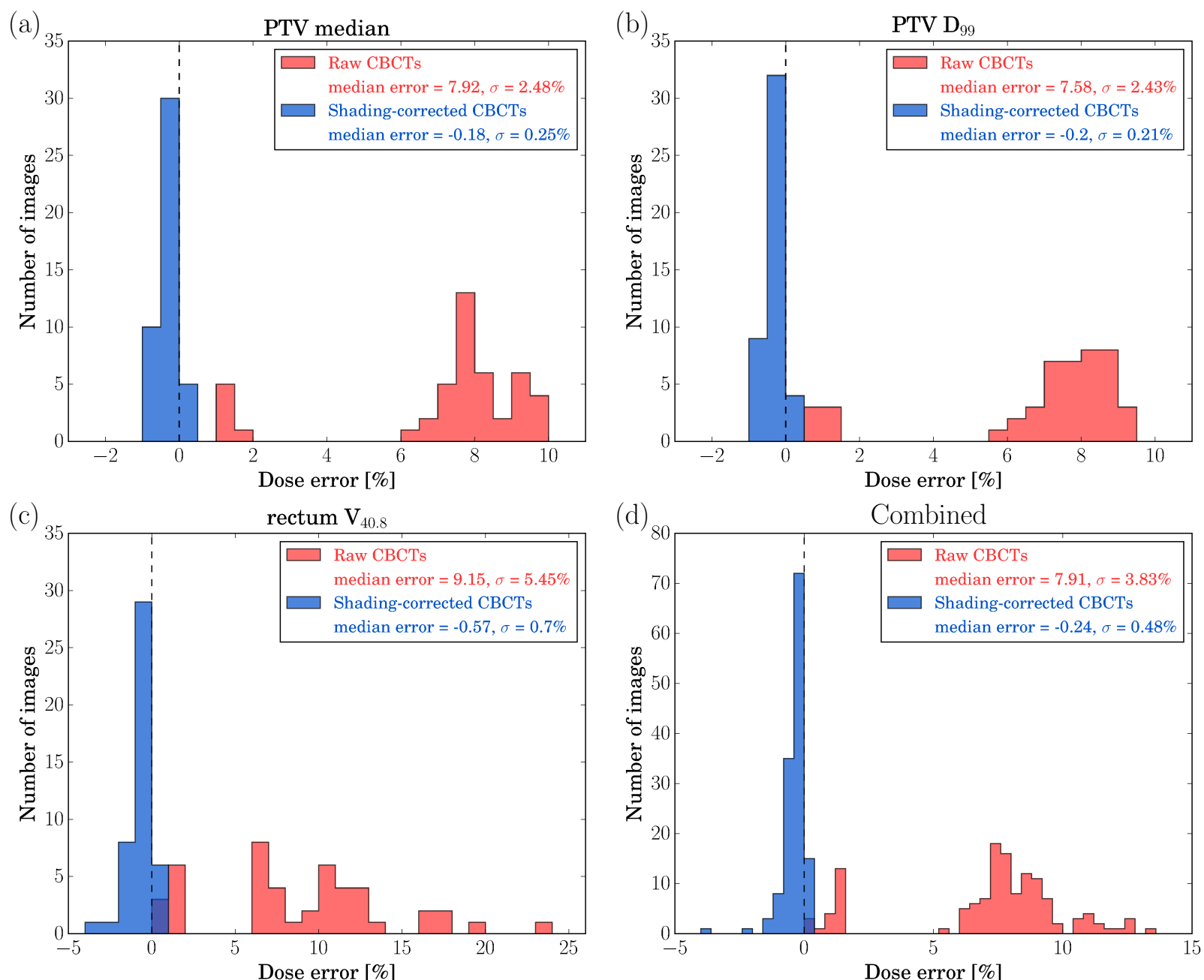

Figure 4. Pelvis CBCT dose errors for raw CBCT (red) and shading corrected CBCT (blue) for (a) PTV median dose (b) PTV $\mathrm{D}_{99}$ (c) rectum $\mathrm{V}_{40.8 \mathrm{~Gy}}$ (d) all metrics combined. 

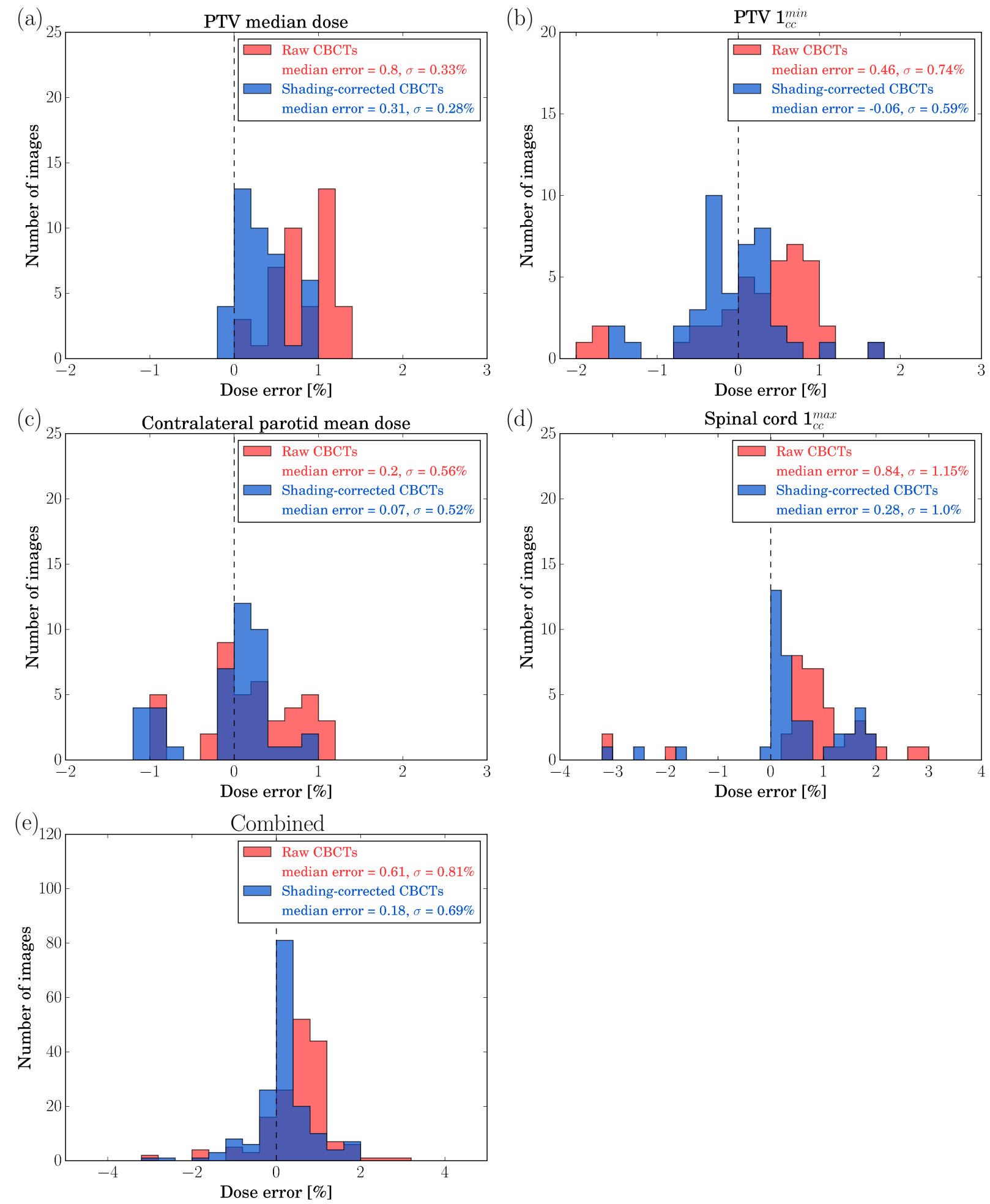

Figure 5. Head and Neck CBCT dose errors for raw CBCT (red) and shading corrected CBCT (blue) for (a) PTV median dose (b) minimum dose to 1cc of PTV (c) contralateral parotid mean dose (d) maximum dose to 1cc of SC (e) all metrics combined. 
Table 3: Summary of results for H\&N dose metrics.

\begin{tabular}{llrrrr}
\hline & Metric & Median err & SD err & Err $<2 \%$ & Err $<3 \%$ \\
\hline \multirow{2}{*}{ Raw } & PTV median & $0.80 \%$ & $0.33 \%$ & $100.0 \%$ & $100.0 \%$ \\
CBCT & PTV 1cc min & $0.46 \%$ & $0.74 \%$ & $100.0 \%$ & $100.0 \%$ \\
& Contralateral parotid mean & $0.20 \%$ & $0.56 \%$ & $100.0 \%$ & $100.0 \%$ \\
& SC 1cc max & $0.84 \%$ & $1.15 \%$ & $88.1 \%$ & $95.2 \%$ \\
& Combined & $0.61 \%$ & $0.81 \%$ & $97.0 \%$ & $98.8 \%$ \\
\hline \multirow{3}{*}{ Corrected } & PTV median & $0.31 \%$ & $0.28 \%$ & $100.0 \%$ & $100.0 \%$ \\
CBCT & PTV 1cc min & $-0.06 \%$ & $0.59 \%$ & $100.0 \%$ & $100.0 \%$ \\
& Contralateral parotid mean & $0.07 \%$ & $0.52 \%$ & $100.0 \%$ & $100.0 \%$ \\
& Co 1cc max & $0.28 \%$ & $1.00 \%$ & $95.2 \%$ & $97.6 \%$ \\
& Combined & $0.18 \%$ & $0.69 \%$ & $98.8 \%$ & $99.4 \%$ \\
\hline
\end{tabular}

\subsection{Lung images}

Figure 6 shows the estimated errors for each of the dose metrics assessed for lung patients. Again the mean error is reduced for shading corrected CBCT. The spread of errors is also reduced, although not as much as for pelvis images. A small proportion of dose metrics for shading corrected lung images have errors greater than $3 \%$. Figure $6 \mathrm{~d}$ shows the combined distribution of dose metric errors for lung CBCT images. The spread of errors is slightly larger than for pelvis and $H \& N$ images, but the dose errors are still significantly improved for corrected CBCT images, with less than $1 \%$ of measurements having error $>5 \%$. Table 4 summarises the median and standard deviation of dose errors for each of the dose metrics used for lung plans. The percentage of dose metric errors greater than $3 \%$ and $5 \%$ is also shown.

Table 4: Summary of results for lung dose metrics.

\begin{tabular}{llrrrr}
\hline & Metric & Median err & SD err & Err $<3 \%$ & Err $<5 \%$ \\
\hline \multirow{2}{*}{ Raw } & PTV median & $4.56 \%$ & $2.07 \%$ & $33.3 \%$ & $64.4 \%$ \\
CBCT & PTV D95 & $4.93 \%$ & $2.18 \%$ & $37.8 \%$ & $51.1 \%$ \\
& SC 1cc max & $4.25 \%$ & $3.25 \%$ & $31.1 \%$ & $62.2 \%$ \\
& Combined & $4.62 \%$ & $2.57 \%$ & $34.1 \%$ & $59.3 \%$ \\
\hline \multirow{3}{*}{ Corrected } & PTV median & $0.04 \%$ & $0.79 \%$ & $97.8 \%$ & $100.0 \%$ \\
CBCT & PTV D95 & $0.03 \%$ & $1.12 \%$ & $97.8 \%$ & $97.8 \%$ \\
& SC 1cc max & $-0.12 \%$ & $1.26 \%$ & $97.8 \%$ & $100.0 \%$ \\
& Combined & $-0.03 \%$ & $1.08 \%$ & $97.8 \%$ & $99.3 \%$ \\
\hline
\end{tabular}

\section{DISCUSSION}

In this work we have demonstrated the clinical utility of CBCT image correction to restore accurate HU values allowing direct use of CBCT for dosimetric assessment of RT delivery. The results show that corrected CBCT can be used to assess the dosimetric effect of anatomical changes occurring during treatment. Practitioners can have a high degree of confidence $(>95 \%)$ that RT dose metrics derived from the corrected CBCT images are accurate to within 2-3\%. This confidence is vital for the adoption into clinical use of corrected CBCT since dose metrics derived from CBCT may be used to make changes to patients radiotherapy (for example if target underdose or OAR overdose is indicated by CBCT assessment).

Key features of our study are:

1. Use of a large set of test images (135 images over 45 patients including 3 anatomic sites). Other studies $^{7,13-16}$ have been based on a relatively small number of images, demonstrating feasibility of CBCT dose calculations rather than measuring performance. 

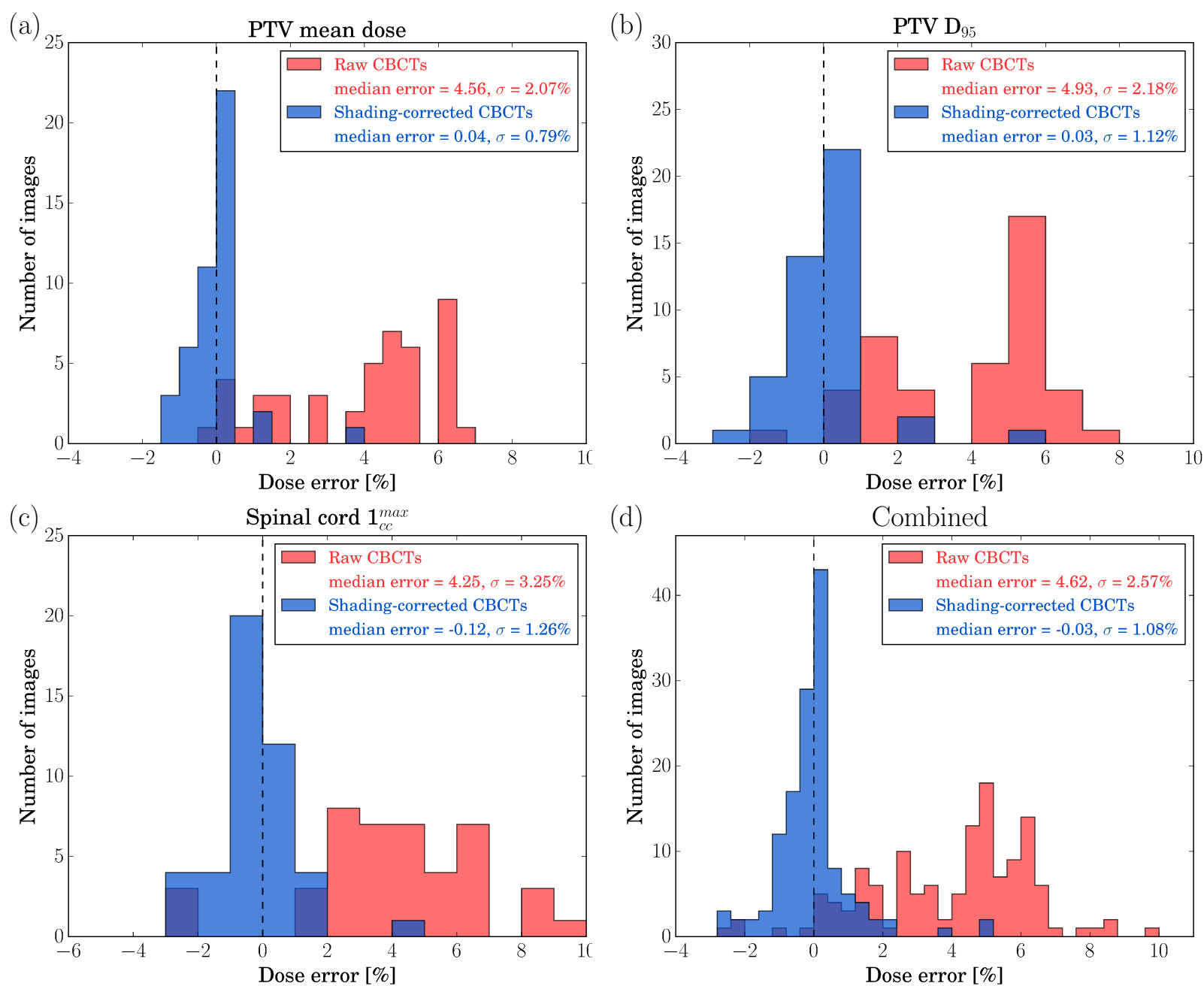

Figure 6. Lung CBCT dose errors for raw CBCT (red) and shading corrected CBCT (blue) for (a) PTV mean dose (b) PTV $D_{95}$ (c) maximum dose to 1cc of SC (d) all metrics combined. 
2. Validation using clinically relevant dose metrics similar to those used in practice to assess accuracy of RT delivery. Previous validation studies of CBCT dose calculations in RT have reported improvement in image-based metrics (e.g. average $\mathrm{HU}$ error ${ }^{6,17}$ ), the clinical effect of which is difficult to interpret.

Dose metric errors for raw $\mathrm{H} \& \mathrm{~N}$ CBCT images (see figure 5) are generally much smaller than those for raw pelvis or lung CBCT. This can be explained firstly because the diameter of the head is smaller than either pelvis or thorax, giving rise to fewer scattered X-rays and consequently less severe scatter artefacts. Secondly the majority of H\&N images included in our study were acquired using a newer version of the XVI software (v5.0) than was used for the pelvis and lung images (mostly XVI v4.5). XVI v5.0 features improved calibration of CBCT Hounsfield Units, which results in generally smaller dose metric errors, arguably good enough for clinical use without further correction. However there is still an improvement after applying the shading correction. This is because the changes in XVI v5.0 only improve the global calibration level of the image and do not correct variations between different areas within the same image. It should also be noted that H\&N CBCT images suffer from significant artefacts below the level of the shoulders, which are also present in XVI 5.0 images, but can be improved by the shading correction. ${ }^{18}$ The effect of these artefacts is not seen in the dose errors presented here because we did not analyse dose in regions of the image where the full patient contour was not within the FOV.

A limitation of our study is that the true CBCT dose distribution is unknown, meaning that we had to approximate it using the override-ratio method described above. Some uncertainty in the estimates of dose metric error remains (as illustrated by our validation study where errors up to $2 \%$ were seen for patients with repeat $\mathrm{CT}$ imaging). Alternative validation methods have been used in other studies, e.g. comparison to CT image acquired on the same day, or deformable image registration. However these alternatives are also subject to uncertainties, often of unknown size. We plan a future study using Monte Carlo to simulate CBCT based on patient CT images. The original CT can then be used to calculate a "gold-standard" dose distribution for comparison to the CBCT-based dose.

An alternative $\mathrm{CBCT}$ dose calculation method is based on deformable image registration of $\mathrm{CBCT}$ to CT images ${ }^{7}$. Deformable image registration tools have recently become available in commercially available software for radiotherapy (e.g. Varian Velocity, Mirada RTx), although their use for dose distributions is still controversial $^{19}$. Further studies are required to assess dose errors arising from geometric uncertainties in the deformable registration process (typically several $\mathrm{mm})^{20}$, and the robustness of deformable registration to large anatomical changes.

The relative simplicity of our correction algorithm allows it to operate robustly. This is an advantage compared to the use of deformable registration (CT-CBCT) which may fail where large changes occur between images. In addition the shading correction operates purely on the final reconstructed CBCT image. No access to projection data or image reconstruction is required. This is an advantage over projection based correction schemes ${ }^{6,10}$, and allows the shading correction to be easily applied to images from different equipment manufacturers.

\section{CONCLUSIONS}

CBCT images processed using our shading correction algorithm can be used to assess RT delivered dose with a high degree of confidence that dose metrics derived from the CBCT dose calculation will be accurate to within $2-3 \%$. The results show clear potential for the algorithm to streamline clinical workflow for use of CBCT in RT, by eliminating the need for time-consuming manual outlining of tissue types for application of density overrides.

\section{Acknowledgments}

This work was supported by the UK Medical Research Council (MRC) grant No. MR/L023059/1. 


\section{References}

[1] McBain, C. A., Henry, A. M., Sykes, J., Amer, A., Marchant, T., Moore, C. M., Davies, J., Stratford, J., McCarthy, C., et al., "X-ray volumetric imaging in image-guided radiotherapy: The new standard in on-treatment imaging," International Journal of Radiation Oncology BiologyPhysics 64(2), 625-634 (2006).

[2] Siewerdsen, J. H., Jaffray, D. A., "Cone-beam computed tomography with a flat-panel imager: Magnitude and effects of x-ray scatter," Med. Phys. 28(2), 220-231 (2001).

[3] Marchant, T. E., Price, G. J., Matuszewski, B. J., Moore, C. J., "Reduction of motion artefacts in on-board cone beam CT by warping of projection images," BJR 84(999), 251-264 (2011).

[4] Mail, N., Moseley, D. J., Siewerdsen, J. H., Jaffray, D. A., "An empirical method for lag correction in cone-beam CT," Med. Phys. 35(11), 5187-5196 (2008).

[5] Zhu, L., Xie, Y., Wang, J., Xing, L., "Scatter correction for cone-beam CT in radiation therapy," Med. Phys. 36(6), 2258-2268 (2009).

[6] Thing, R. S., Bernchou, U., Mainegra-Hing, E., Hansen, O., Brink, C., "Hounsfield unit recovery in clinical cone beam CT images of the thorax acquired for image guided radiation therapy," Phys. Med. Biol. 61(15), 5781 (2016).

[7] Yang, Y., Schreibmann, E., Li, T., Wang, C., Xing, L., "Evaluation of on-board kV cone beam CT (CBCT)-based dose calculation," Phys. Med. Biol. 52(3), 685 (2007).

[8] Veiga, C., Alshaikhi, J., Amos, R., Loureno, A. M., Modat, M., Ourselin, S., Royle, G., McClelland, J. R., "Cone-Beam Computed Tomography and Deformable Registration-Based "Dose of the Day" Calculations for Adaptive Proton Therapy," International Journal of Particle Therapy 2(2), 404-414 (2015).

[9] Moteabbed, M., Sharp, G. C., Wang, Y., Trofimov, A., Efstathiou, J. A., Lu, H.-M., "Validation of a deformable image registration technique for cone beam CT-based dose verification," Med. Phys. 42(1), 196-205 (2015).

[10] Niu, T., Sun, M., Star-Lack, J., Gao, H., Fan, Q., Zhu, L., "Shading correction for on-board cone-beam CT in radiation therapy using planning MDCT images," Med. Phys. 37(10), 5395-5406 (2010).

[11] Fan, Q., Lu, B., Park, J. C., Niu, T., Li, J. G., Liu, C., Zhu, L., "Image-domain shading correction for cone-beam CT without prior patient information," Journal of Applied Clinical Medical Physics 16(6) (2015).

[12] Marchant, T. E., Moore, C. J., Rowbottom, C. G., MacKay, R. I., Williams, P. C., "Shading correction algorithm for improvement of cone-beam CT images in radiotherapy," Phys. Med. Biol. 53(20), 5719 (2008).

[13] Yoo, S., Yin, F.-F., "Dosimetric feasibility of cone-beam CT-based treatment planning compared to CT-based treatment planning," International Journal of Radiation Oncology BiologyPhysics 66(5), 1553-1561 (2006).

[14] Poludniowski, G. G., Evans, P. M., Webb, S., "Cone Beam Computed Tomography Number Errors and Consequences for Radiotherapy Planning: An Investigation of Correction Methods," International Journal of Radiation OncologyBiologyPhysics 84(1), e109-e114 (2012).

[15] Onozato, Y., Kadoya, N., Fujita, Y., Arai, K., Dobashi, S., Takeda, K., Kishi, K., Umezawa, R., Matsushita, H., et al., "Evaluation of On-Board kV Cone Beam Computed Tomography-Based Dose Calculation With Deformable Image Registration Using Hounsfield Unit Modifications," International Journal of Radiation Oncology BiologyPhysics 89(2), 416-423 (2014).

[16] Dunlop, A., McQuaid, D., Nill, S., Murray, J., Poludniowski, G., Hansen, V. N., Bhide, S., Nutting, C., Harrington, K., et al., "Comparison of CT number calibration techniques for CBCT-based dose calculation," Strahlenther Onkol 191(12), 970-978 (2015).

[17] Tsui, T., Wei, J., Zhu, L., "Clinical Validations of Shading Correction for Cone-Beam CT Using Planning CT as a Prior," Med. Phys. 42(6), 3219-3219 (2015).

[18] Joshi, K., Marchant, T., Moore, C., "Shading correction algorithm for cone-beam CT in radiotherapy: Extensive clinical validation of image quality improvement," proc. SPIE, Medical Imaging 2017: Physics of Medical Imaging (2017).

[19] Schultheiss, T. E., Tome, W. A., Orton, C. G., "It is not appropriate to "deform" dose along with deformable image registration in adaptive radiotherapy," Med. Phys. 39(11), 6531-6533 (2012).

[20] Brock, K. K., "Results of a Multi-Institution Deformable Registration Accuracy Study (MIDRAS)," International Journal of Radiation Oncology BiologyPhysics 76(2), 583-596 (2010). 